\title{
Role of a native oxide on femtosecond laser interaction with silicon (100) near the damage threshold
}

\author{
Joel P. McDonald ${ }^{\text {a) }}$ and Arthur A. McClelland \\ Applied Physics Program, University of Michigan, 500 East University Avenue, Ann Arbor, \\ Michigan 48109-1120 \\ Yoosuf N. Picard and Steven M. Yalisove \\ Department of Materials Science and Engineering, University of Michigan, 2300 Hayward Street, \\ Ann Arbor, Michigan 48109-2136
}

(Received 20 December 2004; accepted 2 May 2005; published online 22 June 2005)

\begin{abstract}
Si (100) with and without a 14-25 $\AA$ thick native oxide was laser machined at grazing incidence using a Ti:sapphire femtosecond pulsed laser under ultrahigh vacuum conditions. The resulting damage feature size and morphology indicate that the presence or absence of the native oxide significantly affects the mechanism for femtosecond laser-induced damage. We propose that a fluence-dependent modification of the oxide by the incident laser pulse must be considered when studying femtosecond laser damage of $\mathrm{Si}(100)$ with a native oxide. Data are also presented that are consistent with a dose-dependent phase transformation in the amorphous oxide. The implications of the native oxide, including relative damage thresholds of the underlying $\mathrm{Si}(100)$ and the role of the oxide in damage morphology are addressed. (C) 2005 American Institute of Physics.
\end{abstract}

[DOI: 10.1063/1.1946916]

Silicon and bulk $\mathrm{SiO}_{2}$ have been frequently used to study femtosecond (fs) laser-induced damage. ${ }^{1-10}$ In the case of Si, a wide body of work has been created that addresses the ablation threshold and the morphology of ablated regions. ${ }^{1,4,9}$ Until recently, ${ }^{9,11}$ the great majority of this work was done in air or under low vacuum (up to $10^{-6}$ Torr), and little attention was paid to the omnipresent native oxide of $\mathrm{Si}$ (100). In order to address the role of the native oxide, we have performed a series of multiple-shot fs laser damage experiments under UHV $\left(1 \times 10^{-10}\right.$ Torr $)$, where the presence or absence of the native oxide could be directly controlled.

Samples with native oxide were prepared with an initial degreasing scrub, followed by acetone, methanol, and deionized water baths. Atomically clean samples were etched with a buffered HF solution prior to insertion into UHV. The resulting surface hydride was removed by heating samples to $900{ }^{\circ} \mathrm{C} .{ }^{12,13}$ Low-energy electron diffraction and Auger electron spectroscopy were performed on the samples prior to laser machining, indicating the removal of native oxide and the formation of a well-ordered $(2 \times 1)+(1 \times 2)$ surface.

All samples were laser machined with a Clark-MXR CPA-2001 Ti:sapphire pulsed laser, which operated at $1 \mathrm{kHz}$ with an average power of $780 \mathrm{~mW}$, a central wavelength of $780 \mathrm{~nm}$, generating pulses $\sim 150$ fs in length. The laser was $p$-polarized and directed onto the samples at grazing incidence, such that the beam made an angle of $75^{\circ}-85^{\circ}$ with the sample normal and produced elliptical ablation features on the sample. The focus beam produced a typical (calculated based on assumed Gaussian beam) elliptical area at focus $2.5-7.5 \times 10^{-4} \mathrm{~cm}^{2}\left( \pm 0.5 \times 10^{-4} \mathrm{~cm}^{2}\right)$, where the error reflects the precision of the incident angle of the laser $\left( \pm 0.5^{\circ}\right)$ and measured beam diameter $( \pm 500 \mu \mathrm{m})$ prior to focusing. Once the samples were machined, they were removed from

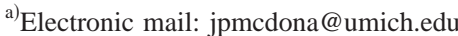

the UHV chamber and studied using a scanning electron microscope (SEM) and an atomic force microscope (AFM). Select samples with native oxide machined in air were studied using optical microscopy (OM).

The SEM images in Fig. 1(b), 1(c), and 1(d) show three fs laser machined features produced with seven shots of varying pulse energy on a sample with surface native oxide, while Fig. 1(e) shows a laser machined feature produced on atomically clean $\mathrm{Si}$ (100) under the same conditions used to produce the feature in Fig. 1(b), with a slightly less fluence. The outermost feature visible in the image [see arrow in Fig. 1(a)] was only found on samples with native oxide. We designate this feature as the outer ring. This ring of damage is located outside the central damage region of the feature and surrounds a region of apparently damage-free material. Diffraction effects have been ruled out as a source for these observed features. The ring feature has been observed on $\mathrm{Si}$ (100) samples laser machined in air under similar conditions for both $s$ - and $p$-polarized laser pulses.

The damage presented in Fig. 1(a) has three distinct surface morphologies that occur in well defined regions. These regions follow the elliptical fluence contours of the incident laser pulse. The feature shown in Fig. 1(c) has two morphologies, while Fig. 1(d) shows only one morphology produced by the laser. The presence of particular damage morphologies is determined by the peak fluence of the incident pulse, and the number of pulses used to produce the feature. The sharpness of the boundaries between different morphologies is consistent with the sharp ablation and modification fluence thresholds observed when using fs pulsed lasers. $^{14}$

The central damage feature in Fig. 1(a) corresponds to the greatest intensity of the Gaussian beam. AFM analysis (not shown for brevity) indicates a rough depression $(\sim 20 \mathrm{~nm})$ signifying the removal of both the native oxide and the underlying Si (100). In this spatial region, we assume that the fluence of the laser pulse is greater than the damage 

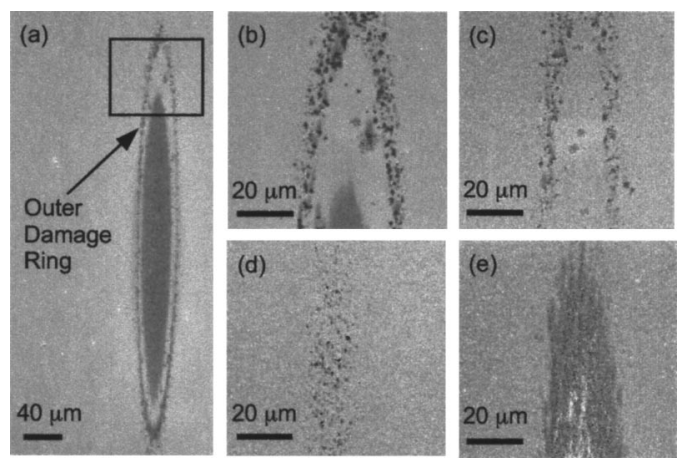

FIG. 1. SEM images of elliptical laser ablation features on Si (100) produced with seven laser pulses at peak fluences shown. (a) Feature produced with peak fluence of $0.320 \mathrm{~J} / \mathrm{cm}^{2}$ showing pitted damage ring surrounding undamaged $\mathrm{Si}$ and a central damage crater. Arrow indicates outer damage ring. (b) Image of inset from (a) showing detail of pitted damage ring, central damage region and intermediate undamaged region. (c) Feature produced with peak fluence of $0.220 \mathrm{~J} / \mathrm{cm}^{2}$ pulses showing only ring of damage surrounding a relatively undamaged region. (d) Feature produced with peak fluence of $0.130 \mathrm{~J} / \mathrm{cm}^{2}$ pulses showing pitted damage region. (e) Feature produced on atomically clean $\mathrm{Si}(100)$ with 7 laser pulses of peak fluence of $0.290 \mathrm{~J} / \mathrm{cm}^{2}$. Notice the absence of any damage ring feature.

threshold of both thin $\mathrm{SiO}_{2}$ and $\mathrm{Si}$ (100). The outer ablation ring is pitted in nature suggesting that the $\mathrm{Si}$ underneath the oxide is ablated and then breaks through the oxide. Similar features have been previously observed and attributed to the relative melting temperatures of $\mathrm{SiO}_{2}$ relative to $\mathrm{Si}(100){ }^{9}$

We suggest that there are three relevant fluence thresholds that are responsible for the observed features. In order of decreasing intensity, these thresholds are the ablation threshold of a thin layer of $\mathrm{SiO}_{2}$, a postulated modification threshold of a thin layer of $\mathrm{SiO}_{2}$, and the damage threshold of bulk $\mathrm{Si}$ (100). Consider the Gaussian fluence spatial profile of the laser pulse on the sample (see Fig. 2). If the fluence at the center of the pulse is greater than the damage threshold of the surface $\mathrm{SiO}_{2}$, we expect the resulting feature to have a central region of damage. As we move radially outward from the center of this pulse, we come to a region on the fluence profile where the fluence drops below the damage threshold of $\mathrm{SiO}_{2}$, however the fluence is still above the modification threshold of the thin $\mathrm{SiO}_{2}$. In some fashion, the modification of the $\mathrm{SiO}_{2}$ decreases the intensity reaching the underlying $\mathrm{Si}$ (100), such that removal of $\mathrm{Si}$ (100) does not occur. The mechanism for this absorption may be a phase change in the $\mathrm{SiO}_{2}$ from one amorphous phase to another. The subdamage threshold modification of $\mathrm{SiO}_{2}$ we observe may be related to the refractive index change used to produce waveguides and gratings under focused fs irradiation in bulk fused silica. ${ }^{15-17}$ As we continue to move away from the center of the shot, the local fluence continues to decrease until it drops below the modification threshold of the $\mathrm{SiO}_{2}$. At this location, the native oxide no longer inhibits the removal of the underlying Si (100), and a ring of pitted features is formed as the underlying $\mathrm{Si}$ (100) explodes from underneath where the fluence exceeds the damage/ablation threshold of the Si (100). We suggest that over a particular range of fluence the native oxide is modified and absorbs the intensity of the laser pulse such that the fluence reaching the underlying $\mathrm{Si}$ (100) surface is insufficient to cause material removal.

In order to understand the laser-induced modification of the native oxide responsible for the observed outer ring, the

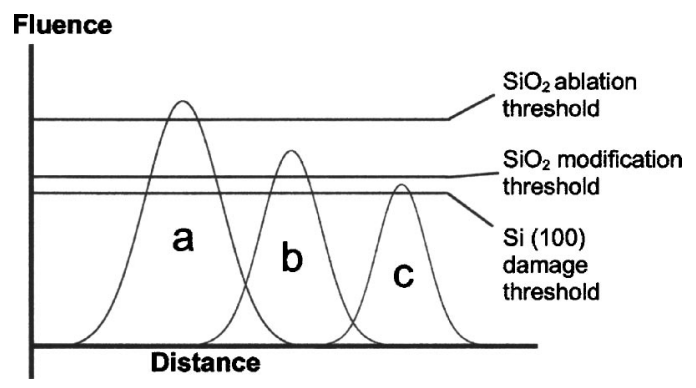

FIG. 2. Gaussian fluence spatial profiles superimposed over relevant fluence thresholds. (a) Peak fluence of pulse at center of pulse greater than damage threshold of $\mathrm{SiO}_{2}$, resulting in features like those made with peak fluence of $0.320 \mathrm{~J} / \mathrm{cm}^{2}$ shown in Fig. 1(a). (b) Peak fluence of pulse less than damage threshold of $\mathrm{SiO}_{2}$ but greater than $\mathrm{SiO}_{2}$ modification threshold, resulting in no material removal at the center of the feature, as in the feature in Fig. 1(c). (c) Peak fluence less than the modification threshold of $\mathrm{SiO}_{2}$, but greater than damage threshold of $\mathrm{Si}(100)$, resulting in a pitted elliptical damage feature, as shown in the SEM image in Fig. 1(d).

area of the undamaged $\mathrm{Si}(100)$ surface between the central damage region and the ablation ring was studied as a function of the number of $p$-polarized laser pulses used to make a particular feature in air (see Fig. 3). As the number of laser pulses increased, the undamaged area decreased as the area of the central damage region increased. After 15 pulses $\left(F_{\text {peak }}=0.280 \mathrm{~J} / \mathrm{cm}^{2}\right)$, at $\sim 5.6^{\circ}$ relative to the surface the undamaged region was no longer apparent on the surface. These results are suggestive of a phase change where nucleation and growth of the new phase continues to completion, allowing light to pass through without further absorption. The presence of the undamaged region for features irradiated in both UHV and air suggests that a process of continued oxide removal is not responsible for the growth of the central damage region with subsequent pulses, as any native oxide removed by the laser is expected to regrow in air (microseconds) well before the arrival of the next pulse (1 ms). Hence, we suggest that the modification of the oxide is a cumulative effect. It was observed that the outer ring appeared after as few as two laser pulses, at which point the area of the undamaged region was maximized. The absence of the ring after a single laser pulse directed at grazing incidence is not at this time well understood and may suggest other absorp-

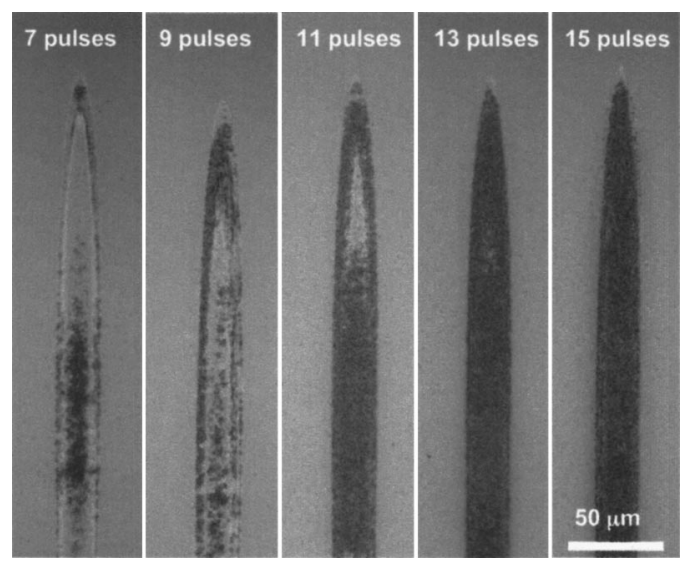

FIG. 3. OM images (in Nomarski mode) of grazing incidence feature morphology as a function of number of laser pulses. Features produced with pulse fluence of $0.280 \mathrm{~J} / \mathrm{cm}^{2}$ in air, with number of pulses shown. Scale is the same for all images. The undamaged region fills as the number of pulses increases, eventually filling in completely after 15 pulses. 
tion mechanisms in the oxide, which we cannot resolve at this time.

The relative damage thresholds for samples with and without native oxide can be addressed by comparing the total area of damage produced by pulses of equal energy. The damage region produced on atomically clean $\mathrm{Si}(100)$ with multiple laser pulses is larger than features produced on $\mathrm{Si}$ (100) with native oxide under the same laser conditions at fluences near the threshold for damage. This implies that the multiple-shot damage threshold of Si (100) can be significantly reduced by removing the native oxide. Calculation of the Fresnel intensity transmission coefficient for light arriving at the silicon substrate at $75^{\circ}-85^{\circ}$ grazing incidence (using bulk refractive index values for $800 \mathrm{~nm}$ light) shows a $20 \%-30 \%$ higher value for atomically clean Si compared to $\mathrm{Si}$ with a native oxide for $p$-polarized light. We observe a slightly larger difference in multishot damage threshold of $55 \pm 30 \%$, where the uncertainty again is related to error in the incident angle measurement $\left( \pm 1.5^{\circ}\right)$ and day-to-day fluctuations in laser stability.

Comparing the ablation threshold values for bulk $\mathrm{SiO}_{2}$ $\left[2-4 \mathrm{~J} / \mathrm{cm}^{2}\right.$ (see Ref. 7)] with the ablation threshold values we observe for the $\mathrm{SiO}_{2}$ thin film, we find the thin film to have an ablation threshold some 10-20 times less than that of the bulk. We argue that the threshold value for a $15-20 \AA$ layer of $\mathrm{SiO}_{2}$ on $\mathrm{Si}$ should be far lower simply because the electrons from the underlying $\mathrm{Si}$ can tunnel into the conduction band of the native oxide and seed the avalanche ionization thought to be responsible for optical breakdown in dielectrics. $^{18}$

In summary, we have demonstrated that the native oxide layer present at the surface of $\mathrm{Si}(100)$ can contribute to damage mechanisms and should be considered when near threshold machining is performed. The ring feature observed on $\mathrm{Si}$ (100) samples laser irradiance at grazing incidence has been explained with a model postulating the presence of three specific thresholds. The observed phenomenon is consistent with a solid-to-solid phase change which saturates upon continued exposure to multiple laser pulses. Additionally, the fs laser damage threshold at grazing incidence is observed to be lower for atomically clean $\mathrm{Si}$ (100) samples than for samples with native oxide. Finally, the ablation threshold of the native oxide is reduced by an order of magnitude relative to bulk $\mathrm{SiO}_{2}$ due to its proximity to the $\mathrm{Si}$ (100) substrate. These results indicate that when performing fs laser damage studies of any material that supports a native oxide, the modification of the oxide must be accounted for when interpreting damage thresholds and morphologies. Additionally, when using standard methods for determining the damage threshold the reflectivity associated with the native oxide must be considered. This work was funded by the NSF (Grant No. DMR03070400) and the DARPA/AFOSF (Grant No. FA9550-04-0136).

${ }^{1}$ J. Bonse, S. Baudach, J. Kruger, W. Kautek, and M. Lenzner, Appl. Phys. A: Mater. Sci. Process. 74, 19 (2002).

${ }^{2}$ H. O. Jeschke, M. E. Garcia, M. Lenzner, J. Bonse, J. Kruger, and W. Kautek, Appl. Surf. Sci. 197-198, 839 (2002).

${ }^{3}$ B. K. A. Ngoi, K. Venkatakrishnan, E. N. L. Lim, B. Tan, and L. H. K. Koh, Opt. Lasers Eng. 35, 361 (2001).

${ }^{4}$ T. H. Her, R. J. Finlay, C. Wu, and E. Mazur, Appl. Phys. A: Mater. Sci. Process. 70, 383 (2000)

${ }^{5}$ T. Q. Jia, Z. Z. Xu, R. X. Li, D. H. Feng, X. X. Li, C. F. Cheng, H. Y. Sun, N. S. Xu, and H. Z. Wang, J. Appl. Phys. 95, 5166 (2004).

${ }^{6}$ C. B. Schaffer, A. Brodeur, and E. Mazur, Meas. Sci. Technol. 12, 1784 (2001).

${ }^{7}$ D. Ashkenasi, M. Lorenz, R. Stoian, and A. Rosenfeld, Appl. Surf. Sci. 150, 101 (1999).

${ }^{8}$ M. Lenzner, J. Kruger, S. Sartania, Z. Cheng, C. Spielmann, G. Mourou, W. Kautek, and F. Krausz, Phys. Rev. Lett. 80, 4076 (1998).

${ }^{9}$ J. Bonse, K. W. Brzezinka, and A. J. Meixner, Appl. Surf. Sci. 221, 215 (2004).

${ }^{10}$ D. Du, X. Liu, G. Korn, J. Squier, and G. Mourou, Appl. Phys. Lett. 64, 3071 (1994).

${ }^{11}$ F. Costache, S. Kouteva-Arguirova, and J. Reif, Solid State Phenom. 9596, 635 (2004).

${ }^{12}$ Y. J. Chabal, G. S. Higashi, K. Raghsvacharik, and V. A. Burrows, J. Vac. Sci. Technol. A 7, 2104 (1989).

${ }^{13}$ Y. J. Chabal, A. L. Harris, K. Raghsvacharik, and J. C. Tully, Int. J. Mod. Phys. B 7, 1031 (1993).

${ }^{14}$ N. Bloembergen, IEEE J. Quantum Electron. QE10, 375 (1974).

${ }^{15}$ J. R. Liu, Z. Y. Zhang, C. Flueraru, X. P. Liu, S. D. Chang, and C. P. Grover, IEEE J. Sel. Top. Quantum Electron. 10, 169 (2004).

${ }^{16}$ J. W. Chan, T. R. Huser, S. H. Risbud, and D. M. J. Krol, Appl. Phys. A: Mater. Sci. Process. 76, 367 (2003).

${ }^{17}$ Y. Kondo, K. Nouchi, T. Mitsuyu, M. Watanabe, and P. G. Kazansky, K. Hirao, Opt. Lett. 24, 646 (1999).

${ }^{18}$ A. P. Joglekar, H. Liu, G. J. Spooner, E. Meyhofer, G. Mourou, and A. J. Hunt, Appl. Phys. B: Lasers Opt. 77, 25 (2003). 